\title{
UV- Ozone Treated Graphene Oxide/ PEDOT:PSS Bilayer as a Novel Hole Transport Layer in Highly Efficient and Stable Organic Solar Cells
}

\author{
Saqib Rafique ${ }^{1}$, Nur Adilah Roslan ${ }^{2,3}$, Shahino Mah Abdullah ${ }^{2}$, Lijie Li ${ }^{1}$, Azzuliani \\ Supangat $^{2}$, Asim Jilani $^{4}$, and Mitsumasa Iwamoto ${ }^{5^{*}}$ \\ ${ }^{1}$ Multidisciplinary Nanotechnology Centre, College of Engineering, Swansea University, \\ Swansea SA1 8EN, United Kingdom \\ ${ }^{2}$ Low Dimensional Materials Research Centre, Department of Physics, Faculty of Science, \\ University of Malaya, 50603 Kuala Lumpur, Malaysia. \\ ${ }^{3}$ Department of Physics, Faculty of Science, Universiti Putra Malaysia, Serdang, 43400 Selangor, \\ Malaysia. \\ ${ }^{4}$ Advanced Membrane Technology Research Centre, Universiti Teknologi Malaysia, 81310 \\ UTM Johor Bahru, Johor, Malaysia \\ ${ }^{5}$ Department of Physical Electronics, Tokyo Institute of Technology, 2-12-1 O-Okayama, \\ Meguro-ku, Tokyo 152-8552, Japan
}

*Corresponding author: iwamoto.m.ac@m.titech.ac.jp 


\begin{abstract}
The utilization of UV-ozone (UVO) treated graphene oxide (GO)/PEDOT:PSS bilayer as hole transport layer (HTL) in solution processed organic solar cells (OSCs) is demonstrated. The HTLs were treated with UVO for $0,5,10$ and $15 \mathrm{~min}$. The $10 \mathrm{~min}$ treated OSC showed the best performance and displayed power conversion efficiency (PCE) of $5.24 \%$, much higher than the untreated OSC device. This enhanced performance is mainly driven by improvements in the short circuit current $\left(\sim 10.82 \mathrm{~mA} / \mathrm{cm}^{2}\right)$ as well as the fill factor $(\sim 57 \%)$ that is ascribed to the moderate reduction of GO and increased work function (WF) of PEDOT:PSS after UVO treatment, which improved the contact conditions between the HTL and photoactive layer. Consequently, extraction efficiency of the photogenerated holes is increased, while recombination probability of holes and electrons in the photoactive layer is decreased. Moreover, the UVO-reduction of GO and consequently increased conductivity of reduced-GO (r-GO) has been modeled and proved using the density functional theory (DFT) simulation. Meanwhile, the 15 min UVO-treated OSC device showed severe reduction in the PCE that dropped to $2.11 \%$, possibly due to couple of factors such as decomposition of chemical bonds, significant increase in the series resistance and pronounced drop in the photovoltaic performance parameters.
\end{abstract}

KEYWORDS:UV-Ozone, reduced graphene oxide, GO/PEDOT:PSS hole transport layer 


\section{INTRODUCTION}

The potential of a low cost, flexible, lightweight and roll-to-roll (R2R) manufacturing process has encouraged researchers to study organic solar cells (OSCs). Among OSCs, bulk heterojunction type OSCs (BHJ OSCs) are one of the most popular ones, where electron donor conjugated polymer is randomly blended with the electron acceptor small molecule fullerene to form the active material [1-3]. In polymer solar cells, the performance parameters such as short circuit current density $\left(\mathrm{J}_{\mathrm{sc}}\right)$, open circuit voltage $\left(\mathrm{V}_{\mathrm{oc}}\right)$ and fill factor $(\mathrm{FF})$ are greatly dependent on the interface electrical properties between the photoactive layer and the electrodes [4]. Therefore, an idea of introducing interfacial layers between the electrodes and photoactive layer has been extensively adopted for the production of high performance and stable OSCs [5, 6]. Hole transport layer (HTL) and electron transport layer (ETL) in BHJ OSCs play numerous roles, including energy level alignments between BHJ and electrode surface, tuning of surface energetics as well as the work function (WF), and restrain recombination to maximize the extraction of charges in OSCs towards the electrodes [7-9].

In this context, poly (3, 4-ethylenedioxythiophene) polystyrene sulfonate or PEDOT:PSS has been extensively used as an HTL due to its appropriate energy levels, excellent transparency and high conductivity $[10,11]$. But, PEDOT:PSS is acidic and hygroscopic that easily causes the device instability [12]. In order to address the stability issues associated with the PEDOT:PSS, several inorganic semiconductors, such as $\mathrm{V}_{2} \mathrm{O}_{5}$ [13], $\mathrm{NiO}$ [14], $\mathrm{WO}_{3}$ and $\mathrm{MoO}_{3}[15,16]$, have been used as an HTL in highly stable OSCs. However, PEDOT:PSS still outperforms its alternatives in terms of efficiency $[17,18]$. By using any of the PEDOT:PSS or its alternatives as an individual HTL material, either the stability or the efficiency has to be compromised. Therefore, 
in recent years, PEDOT:PSS is successfully combined with its alternatives as a hybrid HTL to extract the synergistic benefits of both materials [19-21].

Although, 2-dimensional carbonaceous materials such as graphene oxide (GO) have been extensively used as HTLs due to their benefits of stable aqueous dispersion [22], unique chemical and electronic structures [23], mechanical flexibility [24], solution processability [25], optical transparency and WF similar to that of PEDOT:PSS [26]. However, cell performance with GO is largely dependent on film thickness due to its electrical insulating properties [27]. Moreover, there is difficulty in uniform coverage of indium tin oxide (ITO) at a time, owing to poor film-forming characteristics intrinsically associated with the large flakes of GO [28]. Ultimately, the device performance with GO based HTLs cannot surpass the PEDOT:PSS [29]. Recently, GO has been used as an under-layer below PEDOT:PSS by several groups including our own one [19, 30]. It could effectively preserve ITO from acid corrosion, ion diffusion, and facilitates the extraction of holes due to well matched WF between PEDOT:PSS and GO $[22,31]$. Thus, the resultant devices could complement the drawbacks of either of the individual materials. However, there is still a room to improve the device performance even in case of GO/PEDOT:PSS bilayer, owing to the insulating characteristics of GO. Although, an insulting GO can be tuned by chemical and thermal treatment into semimetal $\mathrm{r}-\mathrm{GO}$ that possess high conductivity and better film quality, thus facilities the charge carriers transport more efficiently $[32,33]$. However, these processes are inadequate for the large-scale production due to toxicity of hydrazine reagent, and ultrahigh-vacuum high temperature requirements, respectively.

To circumvent the aforementioned issues, this work demonstrates an effective approach of UV- ozone (UVO) treated GO/PEDOT:PSS bilayer to function as HTL in BHJ OSCs. Although, there are several reports available for UVO-treated PEDOT:PSS as well as metal oxides and our 
recent work demonstrated the GO also, as single HTL materials [34-36]. However, to the best of our knowledge, there is no work reported so far, for the UVO treated GO/PEDOT:PSS bilayers (HTLs) for BHJ OSCs. For instance, Su et al. [37] and Meng et al. [36] successfully demonstrated the OSCs based on the UVO-treated PEDOT:PSS HTLs and reported a remarkable increase in the power conversion efficiency (PCE) as compared to the untreated PEDOT:PSS. The improved performance was attributed to the increased WF of the PEDOT:PSS. Such treatments increase $V_{o c}$ and enhance the extraction of holes facilitated by the reduction in the energy level offset between the HTL and the photoactive layer. In our recent investigation by Saqib et al. [38] and the work demonstrated by Yingdong et al. [39], significant improvement in the performance of OSCs was recorded with UVO-treated GO based HTLs and the enhanced performance was ascribed to the moderate reduction of GO. Moreover, the resultant device also exhibited significantly better stability. Therefore, the synergistic hybridization of UVO-treated GO and PEDOT:PSS in a GO/PEDOT:PSS bilayer architecture introduced in the present study paves a novel way towards a remarkable improvement in the performance of OSCs, in terms of efficiency, reproducibility and stability, which will ultimately lead towards the R2R manufacturing of OSCs.

\section{EXPERIMENTAL SECTION}

\subsection{Preparation of GO Aqueous Solution}

GO was prepared by the simplified hummers method and the details can be referred to our previous work [30]. The concentration of GO in deionized (DI) water has already been optimized in our previous work to yield best performance of the OSCs [40]. In brief, the GO solution was prepared by dispersing GO into the DI water at the concentration of $1 \mathrm{mg} / \mathrm{mL}$. Dispersion was 
carried out by ultrasonication for few minutes, followed by vigorous stirring over several hours to form a homogeneous mixture.

\subsection{Device Fabrication}

The normal architecture solar cells were fabricated on pre-patterned ITO coated glass substrates provided by Ossila Ltd., United Kingdom with a sheet resistance of $15 \Omega /$ sq. The size of the substrates used was $20 \times 15 \mathrm{~mm}$, with six active pixels of $4.5 \mathrm{~mm}^{2}$ on each. A total of 12 fabrication cycles were repeated for each experimental condition to ensure the repeatability and reproducibility of the fabricated OSCs. The substrates were cleaned by sequential sonication with acetone, isopropyl alcohol and de-ionized water for $20 \mathrm{~min}$ each, followed by oxygen plasma ashing. The substrates were also washed primarily with soap prior to the sonication.

\subsubsection{Deposition and UVO Treatment of HTLs}

Both GO and PEDOT:PSS (PH1000, H.C. Starck) were sequentially spin-coated onto ITO at $4000 \mathrm{rpm}$ for $1 \mathrm{~min}$ in air to form GO/PEDOT:PSS bilayer as an HTL. The PEDOT:PSS solution was also filtered by using $0.45 \mu \mathrm{m}$ PTFE filters (Whatman, Germany) prior to spin coating. In the GO/PEDOT:PSS HTL structure, GO was first exposed to UVO for 0, 5, 10 and 15 min followed by deposition of PEDOT:PSS and further UVO exposure of PEDOT:PSS surface on top of GO/PEDOT:PSS bilayerfor $0,5,10$ and $15 \mathrm{~min}$, respectively. In that latter case the GO surface will probably be exposed twice, however, it is believed that UVO treatment generally effects on the surface of the upper layer with little effect on the underneath GO layer, which will even further reduce the GO (desired). Prior to treatment of PEDOT:PSS, both layers were annealed at $150{ }^{\circ} \mathrm{C}$ for $5 \mathrm{~min}$ to remove absorbed moisture. 


\subsubsection{PCDTBT:PC 71 BMBased Device Fabrication With UVO Treated HTLs}

The polymer used as electron donor segment of the active layer for this study was poly [N9'-heptadecanyl-2,7carbazole-alt-5,5-(4', 7 '-di-2-thienyl-2' ', 1,3'-othiadiazole)], known as PCDTBT. The electron acceptor used was $(6,6)$-Phenyl C71 butyric acid methyl ester or PC $71 \mathrm{BM}$ purchased from Lumtec, Taiwan. The photoactive layer was dissolved in a PCDTBT:PC 71 BM ratio of $1: 4$ at a concentration of $10 \mathrm{mg} / \mathrm{mL}$ in chloroform.

The architecture of devices was ITO/GO/PEDOT:PSS/PCDTBT:PC ${ }_{71} \mathrm{BM} / \mathrm{Al}$, as illustrated in Figure 1. The PCDTBT:PC $71 \mathrm{BM}$ photoactive layer was spin-coated onto the HTLs in a nitrogen filled glovebox at $2000 \mathrm{rpm}$ for $40 \mathrm{sec}$ to form a layer of $\sim 70 \mathrm{~nm}$ thickness. Further, the HTL and the photoactive layer were partially wiped off the cathode-ITO contact using chloroform and a cotton bud. The aluminum (Al) cathode was thermally evaporated in vacuum $\left(<10^{-6}\right.$ Torr $)$ for a desired thickness of $100 \mathrm{~nm}$. Finally, the devices were encapsulated with glass slides using UV-curable epoxy for the characterizations in the air.

\subsection{Device Characterization}

The photovoltaic performance of the OSCs was measured using a Keithley 236 (Keithley Co.) source measurement unit under an Air Mass 1.5 Global (AM 1.5 G) solar simulator with an irradiation intensity of $100 \mathrm{~mW} / \mathrm{cm}^{2}$. The light intensity was calibrated using a Newport power meter 1918-R with calibrated Si-detector 818-UV.

\subsection{Thin Films Characterization}

The transmittance of UVO treated GO/PEDOT:PSS films coated on ITO-glass substrates were recorded with Perkin Elmer UV-visible diffuse reflectance spectrophotometer (Lambda 650) in the range of 300-800 $\mathrm{nm}$. The surface morphology was analyzed by atomic force microscopy (AFM) using tapping mode of Agilent Technologies 5500 Scanning Probe Microscope. X-ray 
photoelectron spectroscopy (XPS) measurements were performed in PHI 5000 Versa Probe II (Ulvac-PHI) using monochromatized $\mathrm{Al} \mathrm{K \alpha}(h v-1486.6 \mathrm{eV})$ anode. XPS data analysis and fitting of Gaussian-Lorentzian line shapes were performed using Multipack software (version 9, ULVAC-PHI, Inc.).

\section{RESULTS AND DISCUSSION}

To explore the effects of the UVO-treatment on the surface of GO and of the PEDOT:PSS layers for the device performance of the GO/PEDOT:PSS HTL, OSCs with the conventional device structure were fabricated by the consecutive coating of the GO/PEDOT:PSS bilayer as an HTL, PCDTBT:PC ${ }_{71} \mathrm{BM}$ blend as a photoactive layer, and $\mathrm{Al}$ cathode on ITO anode, as shown in Figure 1. The experimental details for the device fabrication have already been described in the Experimental Section. GO is a graphene sheet functionalized with oxygen groups in the form of epoxy and hydroxyl groups on the basal plane, and various other types at the edges [24, 41]. Despite its insulating properties, GO is known to be an efficient HTL material and also possesses enhanced stability, primarily due to its WF similar to PEDOT:PSS as well as it preserves ITO from acid corrosion of PEDOT:PSS [25, 26]. Its residual $\mathrm{sp}^{2}$ clusters allow the hole transport via hoping. Similarly, PEDOT:PSS is a benchmark HTL material extensively used in OSCs. The UVO treatment on the surfaces of GO and PEDOT:PSS layers of GO/PEDOT:PSS HTL is expected to significantly enhance the performance of OSCs due to synergistic effects of improved properties of the individual materials brought by the UVO exposure such as increased WF of PEDOT:PSS and moderate reduction of GO $[39,42]$. Further, the moderate reduction and consequently, the increased conductivity of r-GO has been qualitatively analyzed and demonstrated by density functional theory (DFT) simulation of GO. The schematic diagram of the current work and the 
device architecture are presented in Figure 1a and b with the energy levels diagram, are shown in Figure 1c, respectively.

(a)

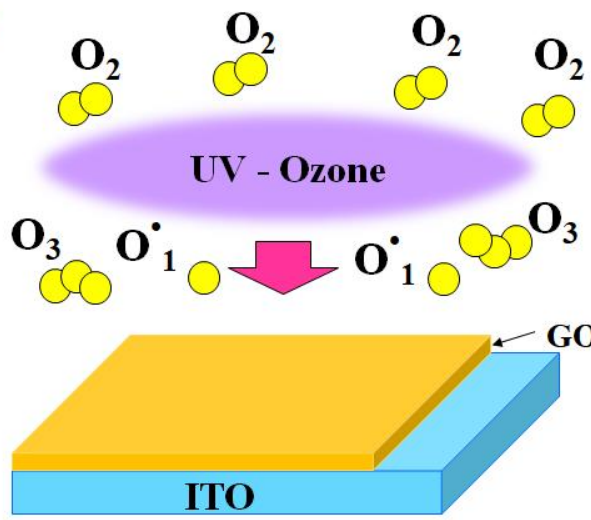

UVO exposure of GO surface

(b)

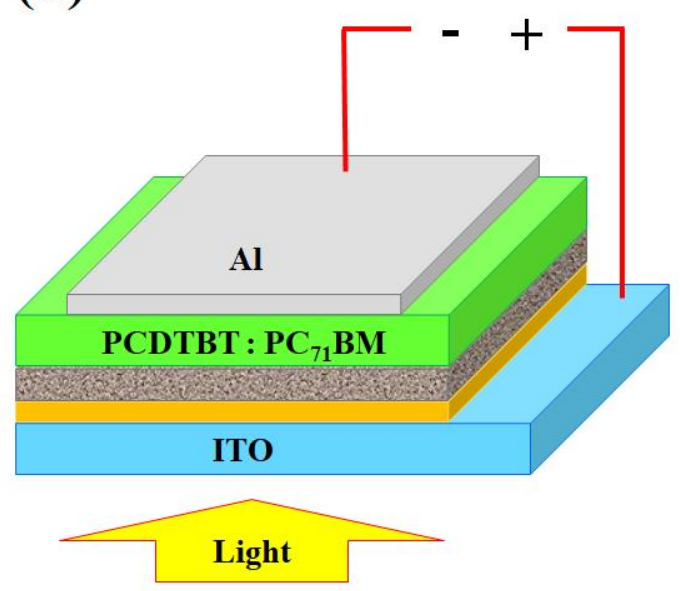

Device Architecture

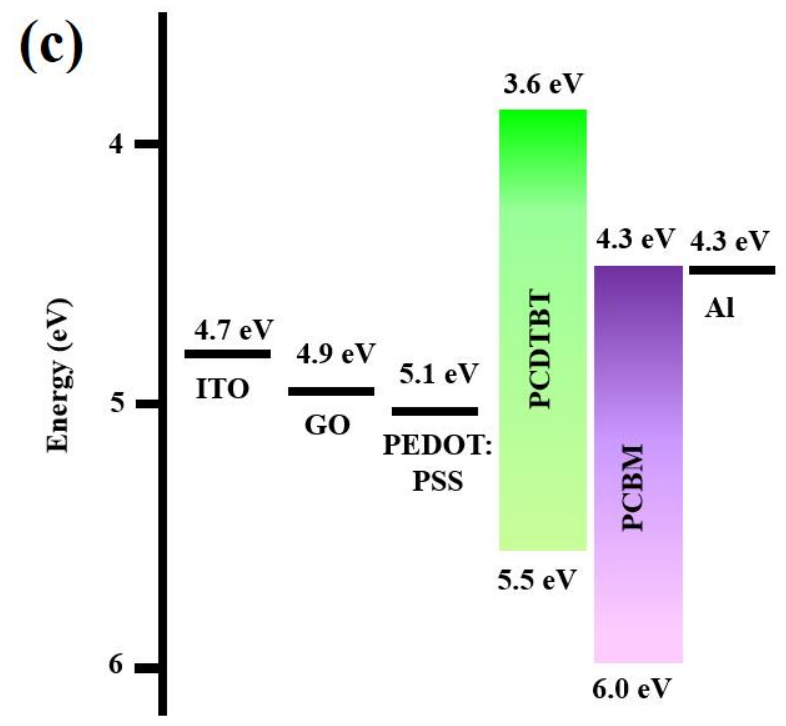

Figure 1: (a) Schematic of the current work showing UVO treatment of GO/PEDOT:PSS HTLs and (b) the device architecture, and (c) energy levels diagram of stacking (energy band of vacuum level alignment for each component in OSC device). 


\subsection{Characterization of UVO Treated Films}

To investigate the properties of HTLs variants, GO/PEDOT:PSS films were replicated on the ITO coated glass substrates following the same deposition parameters as that of the device. Optical and chemical changes were recorded using XPS and UV-vis spectroscopy. The XPS highresolution spectra on both GO single layer and GO/PEDOT:PSS bilayer were recorded to study the chemical changes occurred due to UVO treatment on each surface.

\subsubsection{Optical and Morphological Characteristics}

The optical transmittance spectra of GO/PEDOT:PSS films with various UVO treatment times, deposited on ITO/glass substrates in Figure 2 reveal that, all of the HTLs are highly transparent in the overall wavelength range with transmittance values reaching up to $90 \%$. Further, it was observed that the transparency of the films slightly increases with the increasing UVO treatment times. The observed increase of transmittance in the overall wavelength range between $300-800 \mathrm{~nm}$ could potentially enhance the photogenerated current $\left(\mathrm{J}_{\mathrm{sc}}\right)$ of the device, as evident in our photovoltaic results [43]. Therefore, it will definitely affect the overall performance of the OSCs. The enhanced transmittance is ascribed to the excellent optical properties of both the constituents of HTLs namely GO and PEDOT:PSS, and several other factors along with the transmittance affect the device performance such as surface morphology, chemical changes at the surface, interfaces, and the series resistance $\left(\mathrm{R}_{\mathrm{s}}\right)$ and shunt resistance $\left(\mathrm{R}_{\mathrm{sh}}\right)$ etc. This fact can be observed in the transmittance spectrum of 15 min treated sample where the corresponding device shows poor photovoltaic performance in spite of its best transmittance among all the devices. 


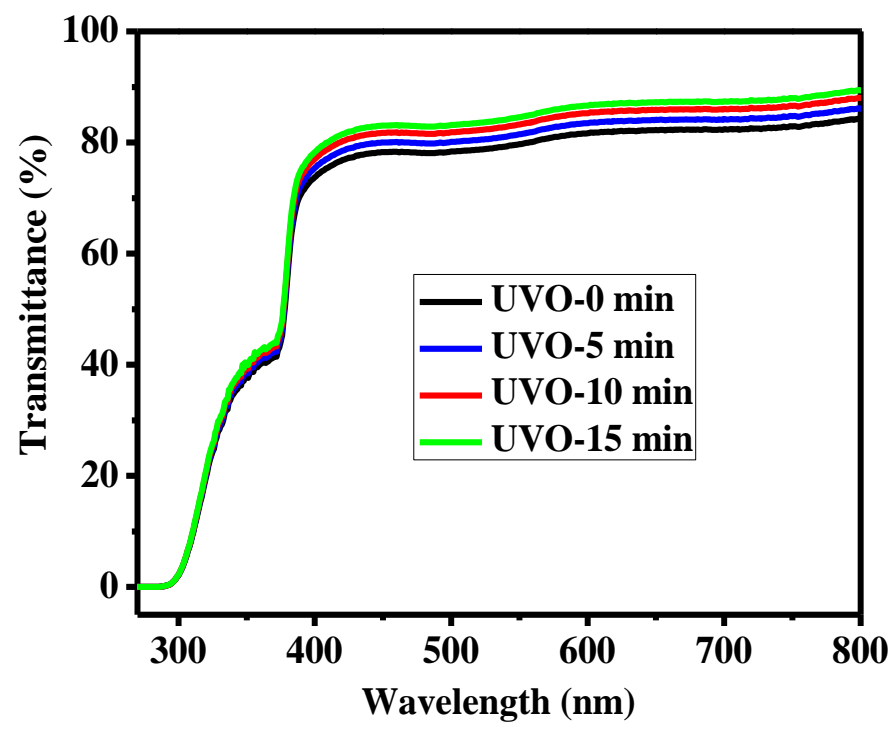

Figure 2: Corresponding optical transmittance spectra of the UVO treated GO/PEDOT:PSS films deposited on ITO/glass substrates.

Generally, the surface morphology of an interfacial layer strongly influences the cell parameters such as the $R_{s}$ and $R_{s h}$ [27]. Since the uniform and fully-covered HTL morphology can induce low $\mathrm{R}_{\mathrm{s}}$ values, thus for the performance improvement in OSCs, it is important to control the quality of the HTL. Therefore, the morphological investigation on top of the GO/PEDOT:PSS film surfaces by AFM gives us valuable information about the surface characteristics of HTLs before and after UVO treatment. An increase in root mean square (RMS) surface roughness was observed for the HTLs deposited on ITO. The morphology of GO/PEDOT:PSS film without UVO treatment is quite smooth with a root mean square (RMS) roughness of $\sim 1.71 \mathrm{~nm}$ in an area of 5 $\mu \mathrm{m} \times 5 \mu \mathrm{m}$, while the RMS roughness increased to $2.28 \mathrm{~nm}$ for $10 \mathrm{~min}$ (best performance) treated film. This finding indicates that UVO treatment does not have significant effect on the morphology of HTL, in good agreement with previously reported work [37]. Moreover, PEDOT:PSS on top of GO can potentially improve the HTL morphology. GO inherently possess rough surface owing to 
its large flakes and deposition of PEDOT:PSS on top of GO in the bilayer structure results in planarizing the irregular GO HTL surface [19]. This indicates that PEDOT:PSS on top of GO can effectively reduce the adverse effects of GO rough morphology and synergistic hybridization of the two materials can potentially enhance the device performance, as evident in our results too.

(a)

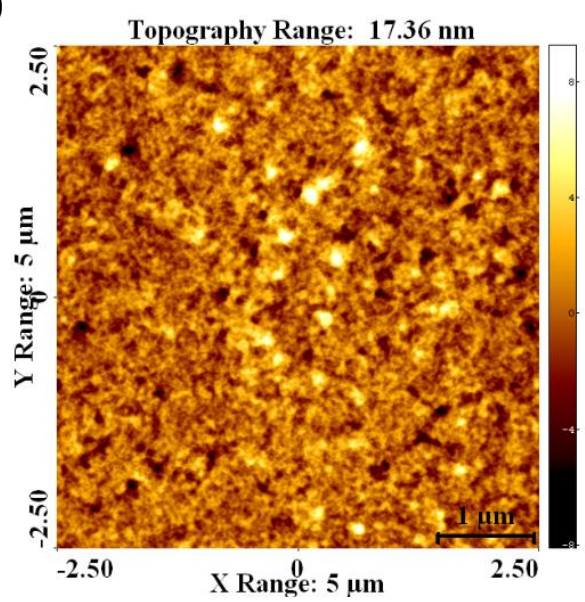

(c)

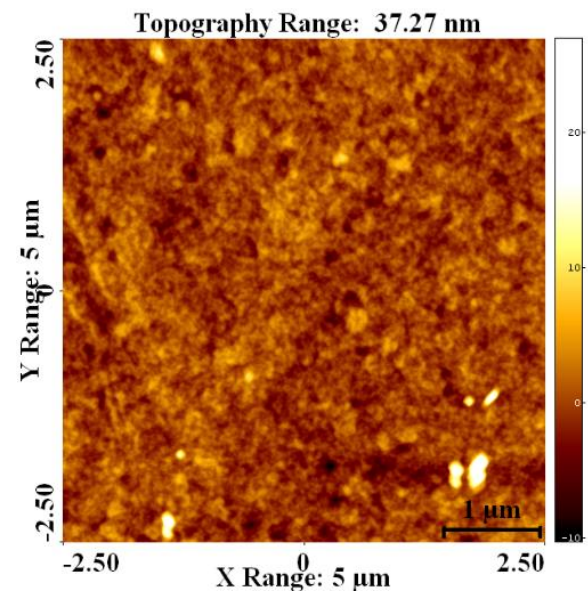

(b)
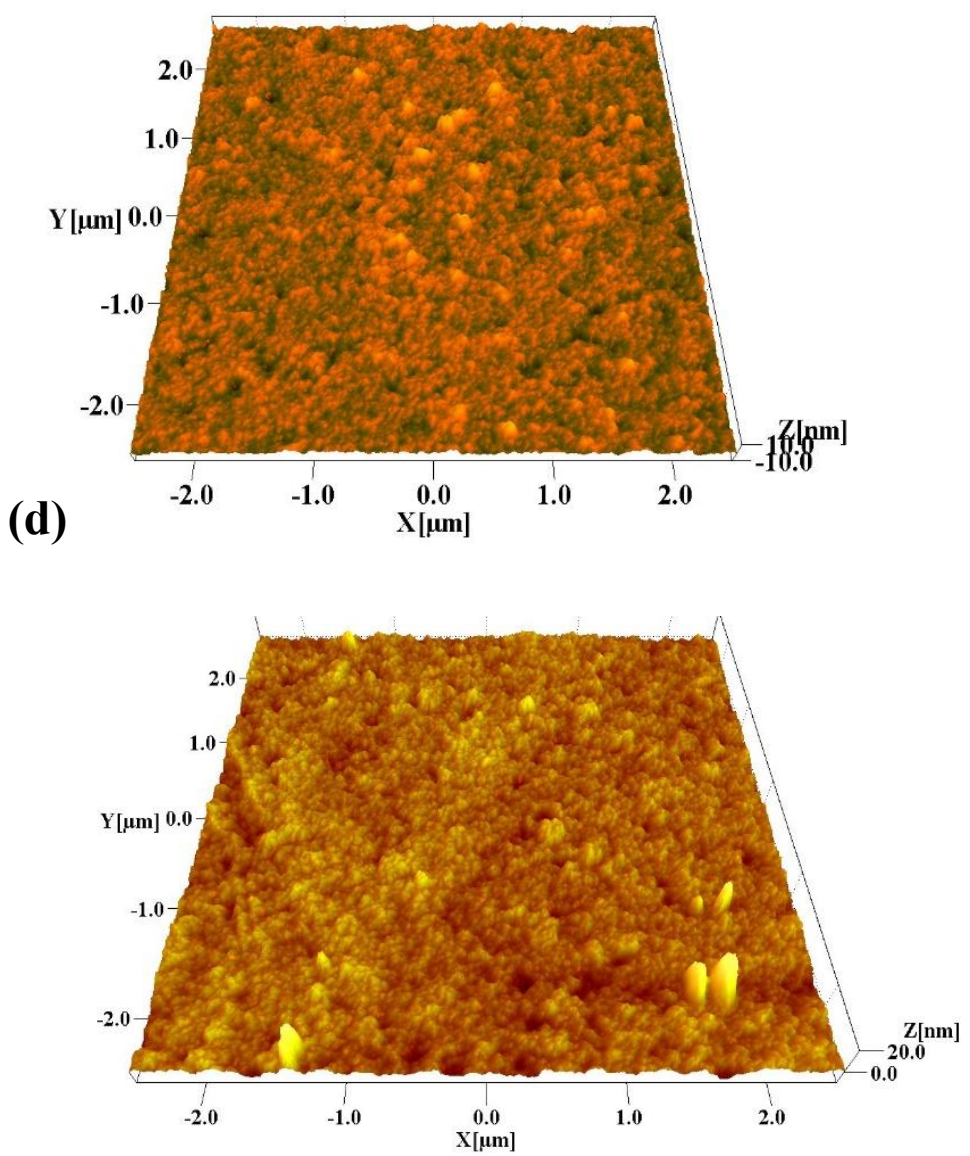

Figure 3: 2D and 3D AFM topography images of GO/PEDOT:PSS films under UVO exposure for $(\mathbf{a}, \mathbf{b}) 0$ and $(\mathbf{c}, \mathbf{d}) 10 \mathrm{~m}$ treated samples. The AFM scan area is $5 \mu \mathrm{m} \times 5 \mu \mathrm{m}$ for GO/PEDOT:PSS films coated on ITO/glass substrates. 


\subsection{XPS Analysis of Chemical Changes Due to UVOTreatment}

\subsubsection{XPS Analysis of Chemical Changes on GO Surface}

In this study, the device with 10 min UVO treatment on the surface of GO and PEDOT:PSS of the GO/PEDOT:PSS HTL showed the best performance with 5.24\% PCE. To confirm the effect of UVO treatment on the chemical properties of GO, XPS analysis was performed on untreated and 10 min treated films as shown in Figure 4a. As mentioned earlier, performance of GO based HTLs is highly sensitive to the thickness of GO due to its insulating characteristics. Reduction/removal of oxygen components from GO can induce transition of GO from electrical insulator to a semimetal [44]. The UVO treatment is expected to effectively reduce the oxygen content in the GO. The $\mathrm{C} 1 \mathrm{~s}$ spectrum of GO before UVO treatment clearly indicates the considerable degree of oxidation, and is deconvoluted into three components that correspond to carbon atoms in different functional groups. The non-oxygenated ring $\mathrm{C}(\mathrm{C}-\mathrm{C}$ bond at $284.65 \mathrm{eV})$ belongs to $\mathrm{sp}^{2}$ carbon [45], the $\mathrm{C}$ in $\mathrm{C}-\mathrm{O}$ bonds at $286.80 \mathrm{eV}$ is attributed to $\mathrm{sp}^{3}$ carbon species [46] and the carbonyl $\mathrm{C}(\mathrm{C}=\mathrm{O})$ at $288.40 \mathrm{eV}$ [47]. After UVO treatment for $10 \mathrm{~min}$, the oxidation species such as $\mathrm{C}-\mathrm{O}$ has significantly reduced while the $\mathrm{C}=\mathrm{O}$ bond has totally disappeared. Moreover, the peak corresponding to the $\mathrm{C}-\mathrm{C}$ bond (to $\mathrm{sp}^{2}$ carbon) was the major feature of $\mathrm{C} 1 \mathrm{~s}$ spectrum after UVO reduction of GO, which is attributed to the significant restoration of conjugated structure of graphene [48]. After reduction, a comparatively low intensity peak corresponding to $\mathrm{C}-\mathrm{C}=\mathrm{C}$ bond has also emerged which clearly indicates the successful reduction of GO [49]. It obviously indicates that oxygen functional groups, mainly responsible for the insulating characteristics of solution processed GO can be effectively removed through the facile UVO treatment. 


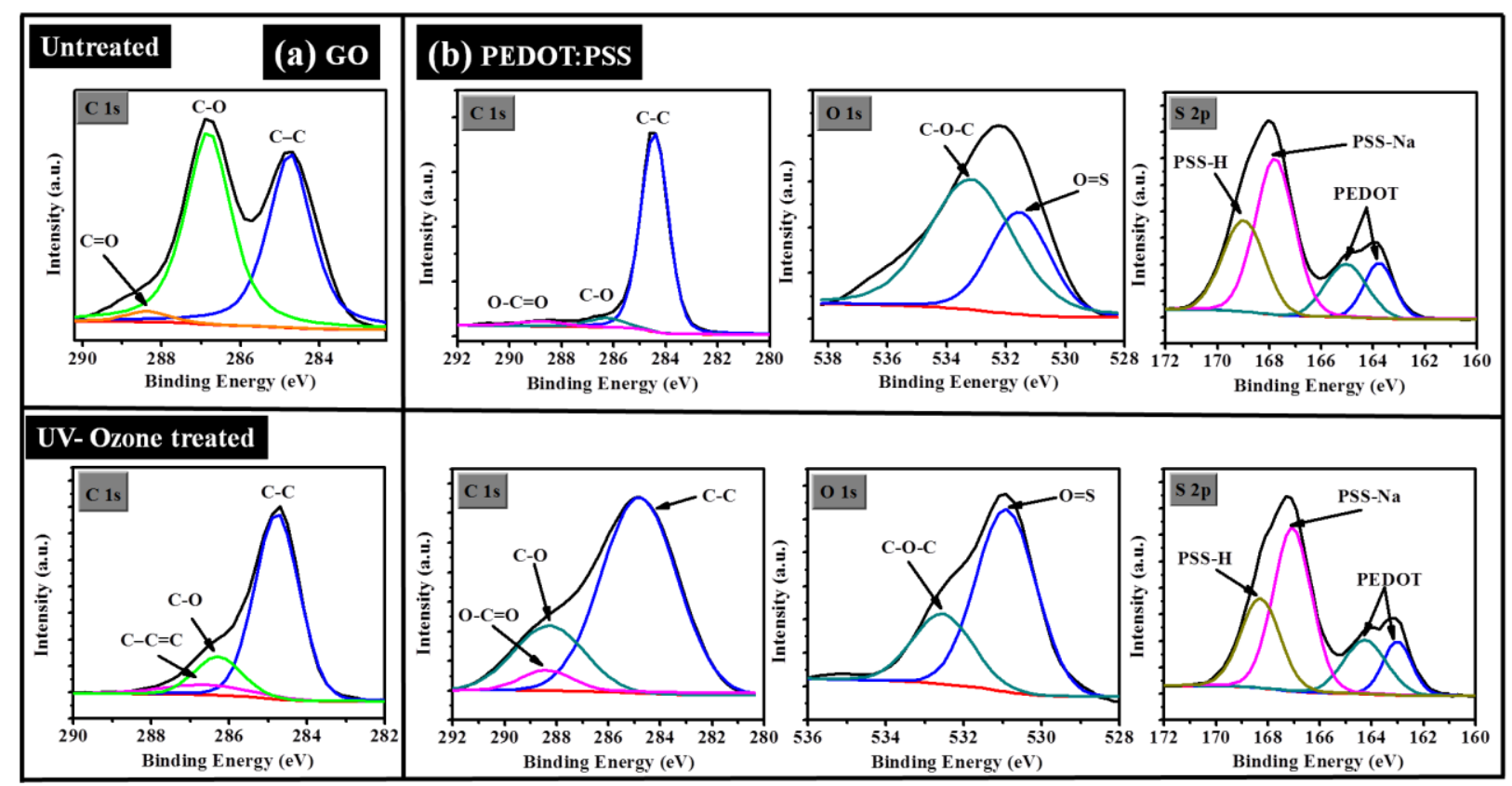

Figure 4: (a) The $\mathrm{C} 1 \mathrm{~s}$ of $\mathrm{GO}$, and (b) $\mathrm{C} 1 \mathrm{~s}, \mathrm{O}$ 1s, and $\mathrm{S}$ 2pcore level spectra of PEDOT:PSS surface of the untreated (upper) and UVO treated (10min) GO/PEDOT:PSS films (below).

\subsubsection{XPS Analysis of Chemical Changes on PEDOT:PSS Surface}

Figure $4 \mathrm{~b}$ shows the C 1s, O1s and S 2p XPS core-level spectra of PEDOT:PSS before and after 10 min UVO treatment. The C1s spectra for the UVO treated sample appear as typical of an oxidized PEDOT:PSS [42, 50]. It indicates a substantial increase in the oxygen containing components, both in the $\mathrm{C}-\mathrm{O}$ (hydroxyl and epoxy group) and the $\mathrm{O}-\mathrm{C}=\mathrm{O}$ (carboxylates) bonds. The $\mathrm{C}=\mathrm{O}$ component has increased from 5.69 to $31.73 \%$, whereas $\mathrm{O}-\mathrm{C}=\mathrm{O}$ has increased from 4.22 to $19.52 \%$ after UVO treatment, consistent with oxidation of polymer backbone $[42,50]$. The O 1s core-level spectra for both the samples (untreated and UVO treated) shown in Figure $4 \mathrm{~b}$ can be assigned as the convolution of two different components, namely C-O-C of the PEDOT centered at $\sim 533 \mathrm{eV}$ and $\mathrm{O}=\mathrm{S}$ of PSS centered at $\sim 531.5 \mathrm{eV}$ [51-53]. It can be observed from the $\mathrm{O} 1 \mathrm{~s}$ spectra, the $\mathrm{O}=\mathrm{S}$ in the sulfonic acid group of PSSH has significantly increased after UVO 
treatment, whereas, the C-O-C component has slightly reduced [54]. The $\mathrm{S} 2 \mathrm{p}$ spectra for PEDOT:PSS in both samples appear in two components. The peaks at lower binding energies corresponding to PEDOT originate from sulphur atoms linked to carbon atoms in the chemical structure of PEDOT:PSS[55, 56]. The component at higher binding energies corresponds to the sulphur atoms in the sulfonic acid PSS polymer [57]. The contribution of PSS to the $S 2 p$ peak is due to the PSS: $\mathrm{H}^{+}$and PSS: $\mathrm{Na}^{+}$doping ions as shown in Figure 4b [53]. No further changes were observed in the S 2p spectrum of the treated sample. However, it was observed that all the core levels have been shifted to the lower binding energies. The binding energies of $\mathrm{C}$ 1s were shifted by almost $0.20 \mathrm{eV}$, whereas, the $\mathrm{O} 1 \mathrm{~s}$ and $\mathrm{S} 2 \mathrm{p}$ spectra experienced a shift of approximately 0.63 and $0.74 \mathrm{eV}$, respectively to the lower binding energies. This indicates an increase in the Fermi level position (i.e., chemical potential) and consequently, the WF of PEDOT:PSS films with UVO treatment has also increased[42, 50].

\subsection{Photovoltaic Characteristics of the OSCs}

The operating characteristics of the OSCs with the UVO treated GO/PEDOT:PSS HTLs in terms of (a) the current density versus applied voltage, (b) the power versus applied voltage, (c) the normalized PCE and, (d) the $\mathrm{R}_{\mathrm{s}}$ are shown in Figure 5. The extracted solar cell performance characteristics are summarized in Table 1. The reference device with untreated GO/PEDOT:PSS HTL presented a $\mathrm{J}_{\mathrm{sc}}$ of $9.40 \mathrm{~mA} / \mathrm{cm}^{2}$, a $\mathrm{V}_{\mathrm{oc}}$ of $0.85 \mathrm{~V}$, a FF of $41 \%$ and a PCE of $3.28 \%$. On the other hand, the OSCs with the 5 and 10 min UVO treatment on the surface of GO and PEDOT:PSS of the GO/PEDOT:PSS HTL showed significantly improved efficiency. In particular, optimized OSCs with the 10 min UVO treatment exhibited remarkably improved PCE of $5.24 \%$ with a $\mathbf{J}_{\mathrm{sc}}$ of $10.82 \mathrm{~mA} / \mathrm{cm}^{2}$, a $\mathrm{V}_{\text {oc }}$ of $0.85 \mathrm{~V}$ and a FF of $57 \%$ followed by the device with the 5 min UVO 
treatment, where the device showed a $\mathrm{J}_{\mathrm{sc}}$ of $10.82 \mathrm{~mA} / \mathrm{cm}^{2}$, a $\mathrm{V}_{\mathrm{oc}}$ of $0.85 \mathrm{~V}$, a FF of $57 \%$ and a PCE of $5.01 \%$. While, the device with the 15 min treatment showed a pronounced drop in PCE mainly due to a significant fall in $\mathrm{J}_{\mathrm{sc}}\left(5.31 \mathrm{~mA} / \mathrm{cm}^{2}\right)$, and comparatively low $\mathrm{V}_{\mathrm{oc}}(0.75 \mathrm{~V})$ and $\mathrm{FF}$ (53\%). The fall in the performance of OSCs for longer UVO exposure is mainly attributed to a

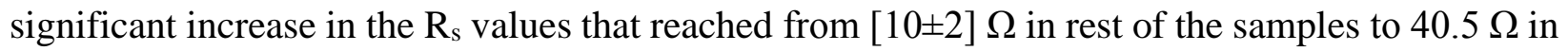
15 min treated sample, as shown in Figure 5d. While, relatively low performance of the untreated device is ascribed to a pronounced reduction in the FF. The optimum exposure time for GO/PEDOT:PSSHTLs for OSCs is $10 \mathrm{~min}$.

(a)

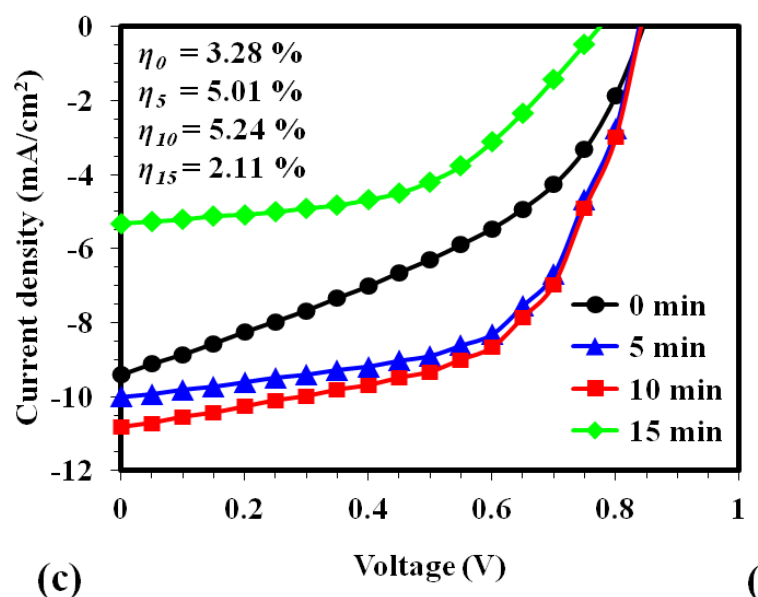

(c)

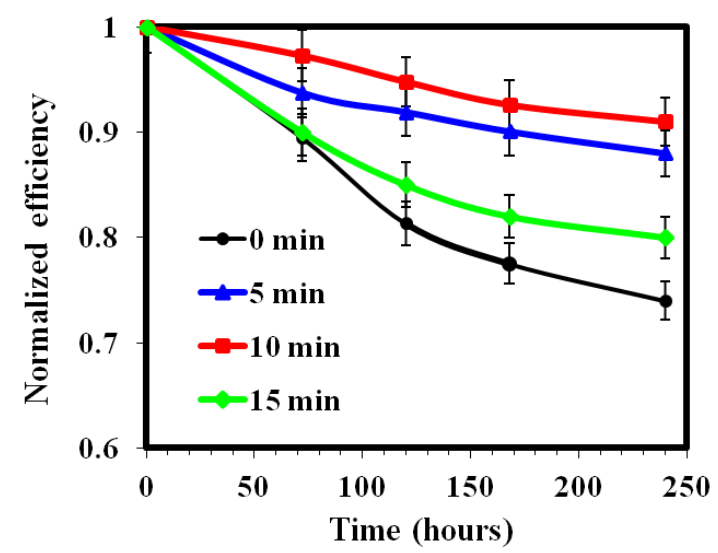

(b)

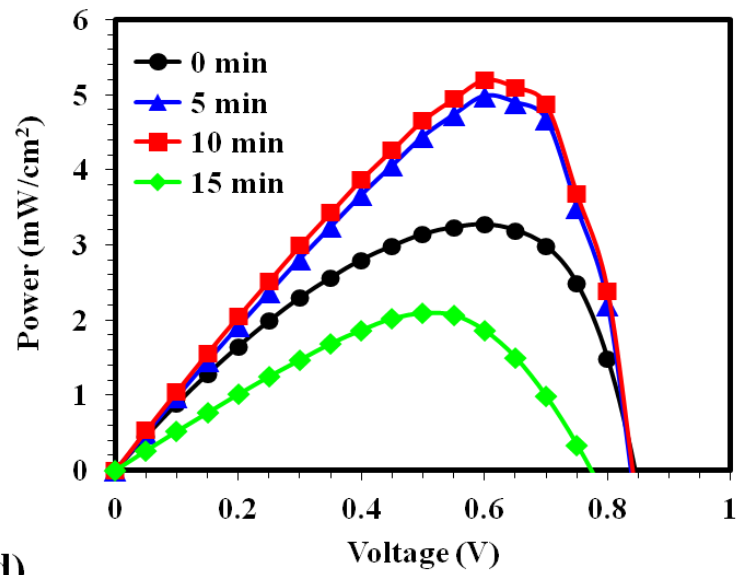

(d)

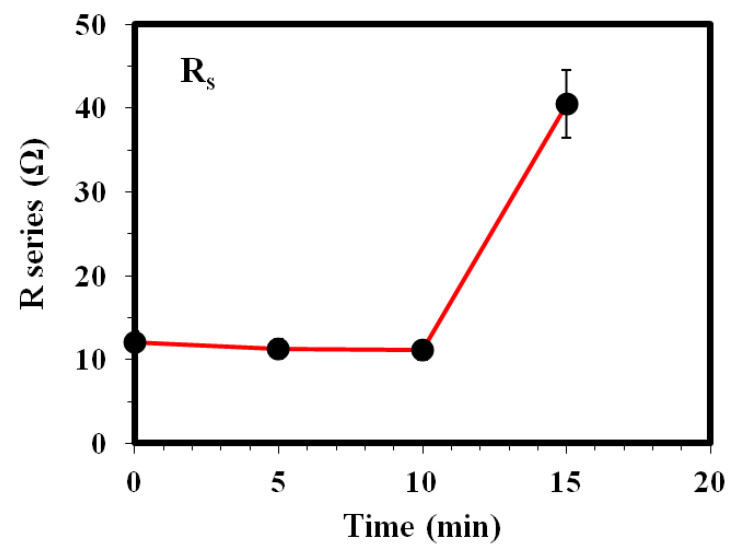


Figure 5: Device characteristics for OSCs with UVO treated GO/PEDOT:PSS HTLs as a function of varied treatment time for $0,5,10$ and $15 \mathrm{~min}$. (a) Current density-voltage $(J-V)$ characteristics and (b) Power vs Voltage plots under AM1.5G solar spectrum with an irradiation intensity of 100 $\mathrm{mW} / \mathrm{cm}^{2}$. (c) Stability plots of OSCs over $240 \mathrm{~h}$. (d) $\mathrm{R}_{\mathrm{s}}$ of the OSCs evaluated from $J V$ curves.

The maximum output power $\left(\mathrm{P}_{\max }\right)$ of the OSC device is increased from $3.3 \mathrm{~mW} / \mathrm{cm}^{2}$ to $5.0 \mathrm{~mW} / \mathrm{cm}^{2}$ after the HTL (GO/PEDOT:PSS) was exposed to the UVO for $5 \mathrm{~min}$. Additional exposure time up to $10 \mathrm{~min}$ led to a slight increase in output power to $5.2 \mathrm{~mW} / \mathrm{cm}^{2}$. However, further treatment of UVO onto HTL for 15 min has resulted in a pronounced reduction in output power $\left[2.1 \mathrm{~mW} / \mathrm{cm}^{2}\right]$, which is even lower than that of the OSC with untreated HTL. It shows that a 10 min UVO exposure onto the HTL is an optimized condition to achieve the highest output power as well as the PCE, as shown in Figure 5b.

To further expand the feasibility and potential of UVO treated GO/PEDOT:PSS HTLs, the lifetime of the devices as a function of exposure time to ambient atmosphere were investigated as shown in Figure 5c. The device with 5 and 10 min UVO treated GO and PEDOT:PSS surfaces showed almost similar behavior and PCE of the devices retained above $90 \%$ of the initial values after $240 \mathrm{~h}$ of ambient exposure. However, the untreated and 15 min treated samples showed more pronounced reduction in the PCE that dropped to almost 80 and $75 \%$ of initial values over $240 \mathrm{~h}$, respectively. The improved stability with the optimized UVO treatment time is attributed to the fact that GO also acts as a barrier against the diffusion of unwanted indium into the HTL and photoactive layer [19]. Moreover, r-GO functions as more stable HTL and also shows better passivation property against oxygen and moisture as compared to PEDOT:PSS [33]. However, further studies are essential to elucidate the mechanism. 
Table 1: PV-characteristics of OSCs with UVO treated GO/PEDOT:PSS HTLs.

\begin{tabular}{l|ccccc}
\hline \multicolumn{1}{c|}{$\begin{array}{c}\text { UVO exposure } \\
\text { time (min ) }\end{array}$} & $\mathbf{J}_{\mathbf{s c}}$ & $\mathbf{V}_{\text {oc }}$ & FF & PCE & $\mathbf{R}_{\mathbf{s}}$ \\
& {$\left[\mathbf{m A} / \mathbf{c m}^{2}\right]$} & {$[\mathbf{V}]$} & {$[\%]$} & {$[\%]$} & {$[\mathbf{\Omega}]$} \\
\hline $\mathbf{0}$ & 9.40 & 0.85 & 41.0 & 3.28 & 12.1 \\
$\mathbf{5}$ & 10.00 & 0.85 & 59.0 & 5.01 & 11.3 \\
$\mathbf{1 0}$ & 10.82 & 0.85 & 57.0 & 5.24 & 11.1 \\
$\mathbf{1 5}$ & 5.31 & 0.75 & 53.0 & 2.11 & 40.5 \\
\hline
\end{tabular}

For further comparison, the device performances was investigated by employing UVO treated single GO and PEDOT:PSS HTLs for 10 min as reference devices, and compared with the UVO treated (10 min) GO/PEDOT:PSS bilayer HTL. Figure 6 shows representative JV curves and the important parameters are summarized in Table 2. All the solar cells parameters have been enhanced by the introduction of GO/PEDOT:PSS HTL as compared to either of the single materials. The cell with the GO/PEDOT:PSS showed a PCE of 5.24\%, a $\mathrm{J}_{\mathrm{sc}} 10.82 \mathrm{~mA} / \mathrm{cm}^{2}$, a $\mathrm{V}_{\mathrm{oc}}$ of $0.85 \mathrm{~V}$ and a FF of $57 \%$ as compared to the single PEDOT:PSS (a PCE of 4.01\%, a $\mathbf{J}_{\mathrm{sc}} 9.90$ $\mathrm{mA} / \mathrm{cm}^{2}, \mathrm{~V}_{\mathrm{oc}}$ of $0.80 \mathrm{~V}$ and a FF of $50.6 \%$ ) or GO (a PCE of $4.42 \%$, a $\mathrm{J}_{\mathrm{sc}} 10.03 \mathrm{~mA} / \mathrm{cm}^{2}$, a $\mathrm{V}_{\text {oc }}$ of $0.84 \mathrm{~V}$ and a FF of $52.5 \%$ ). The improved performance is mainly attributed to the pronounced increase in $\mathrm{V}_{\mathrm{oc}}$ and $\mathrm{FF}$ as well as a considerable increase in the $\mathrm{J}_{\mathrm{sc}}$. A detailed comparative analysis on the performance improvement of GO/PEDOT:PSS bilayer HTL over the single GO or PEDOT:PSS HTLs have been drawn in the Section 3.5. 
(a)

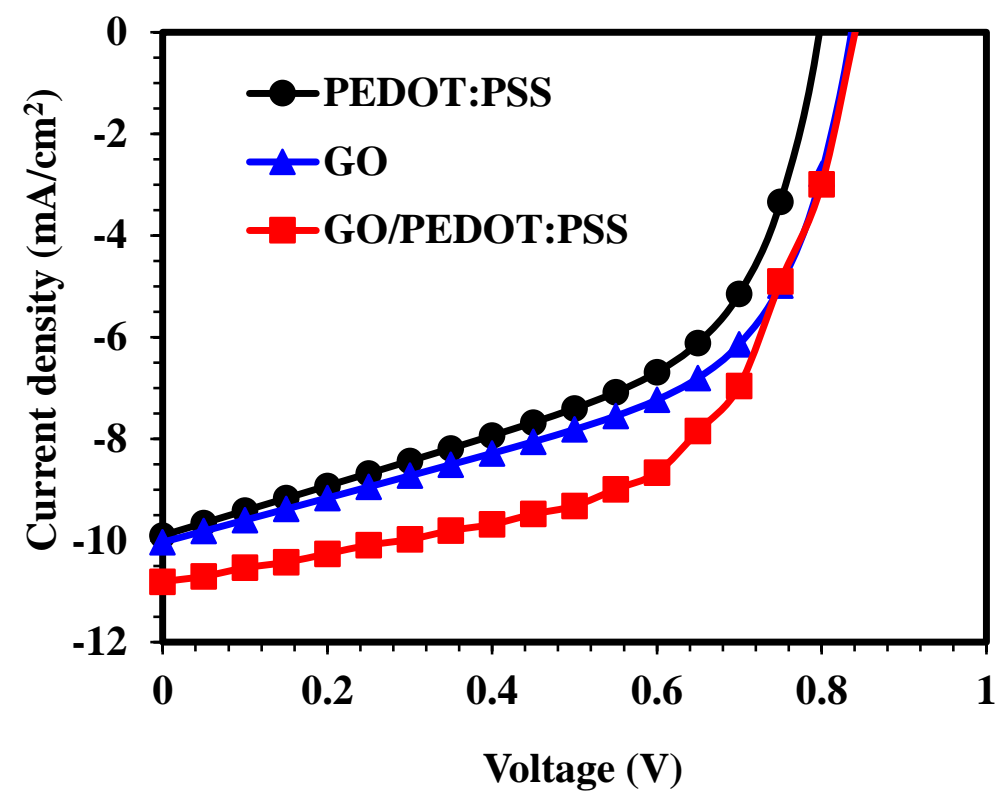

(b)

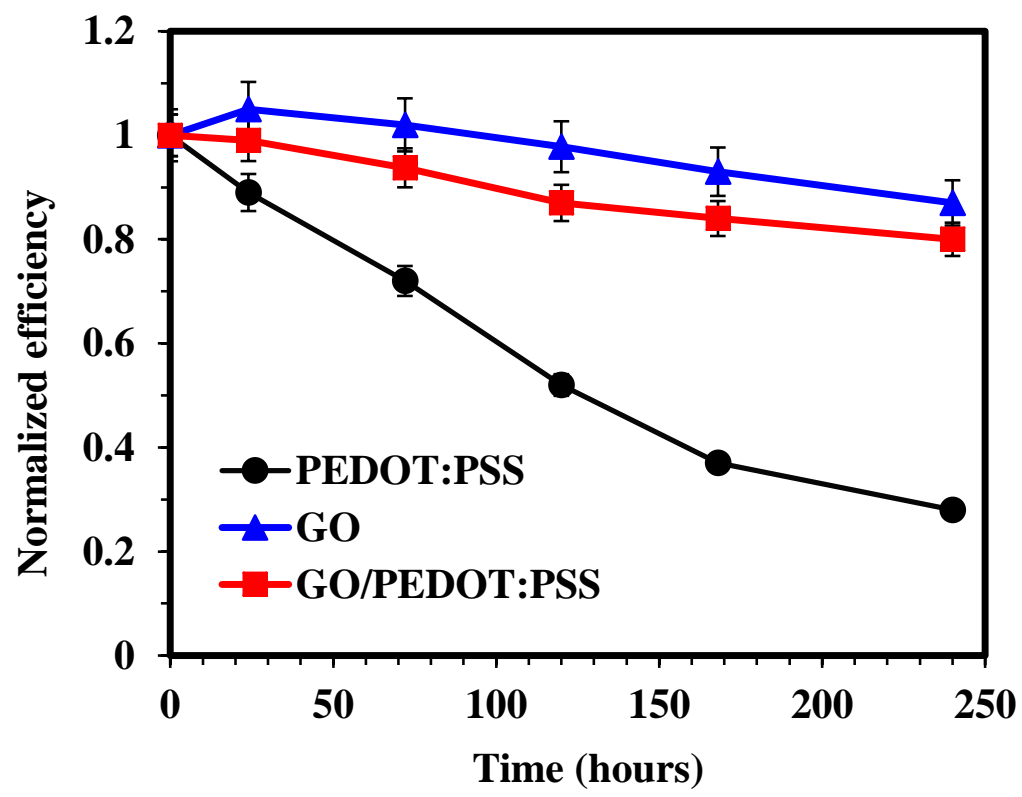

Figure 6: (a) Device performance comparison between UVO treated (for $10 \mathrm{~min}$ ) GO and PEDOT:PSS as reference HTLs with GO/PEDOT:PSS bilayer HTL. (b) Decay in PCE of a 
GO/PEDOT:PSS based OSCs and an OSC with the UVO-GO and UVO-PEDOT:PSS HTLs over the period of $240 \mathrm{hrs}$.

Figure 6b compares the life-time of the devices with the UVO treated single GO and PEDOT:PSS with that of GO/PEDOT:PSS bilayer, and the cell performance was recorded as the function of exposure time in air. The PCE of the PEDOT:PSS based device showed a sharp decline, while the stability of the devices was significantly improved by inserting the GO/PEDOT:PSS bilayer or GO HTL. The device consisting of UVO treated GO only HTL retained $87 \%$ of its efficiency after 240 hrs followed by GO/PEDOT:PSS where it retained around $80 \%$ of its initial efficiency. The improved stability of GO/PEDOT:PSS HTL is expected because GO prevents the direct contact of acidic PEDOT:PSS with the ITO [19]. Consequently, the OSCs using UVO treated GO/PEDOT:PSS showed remarkable efficiency and stability.

Table 2: Summary of the device performance comparison between UVO treated single PEDOT:PSS, GO and bilayer GO/PEDOT:PSS HTL for 10 min (best optimum time).

\begin{tabular}{c|cccc}
\hline $\begin{array}{c}\text { UVO Treated } \\
\text { HTLs }\end{array}$ & $\mathbf{J}_{\mathbf{s c}}$ & $\mathbf{V}_{\text {oc }}$ & FF & PCE \\
{$\left[\mathbf{m A} / \mathbf{c m}^{2}\right]$} & {$[\mathbf{V}]$} & {$[\%]$} & {$[\%]$} \\
\hline PEDOT:PSS & 9.90 & 0.80 & 50.6 & 4.01 \\
GO & 10.03 & 0.84 & 52.5 & 4.42 \\
\hline
\end{tabular}

Furthermore, as shown in Figure 7, highly reproducible device performances were realized using UVO treated GO/PEDOT:PSS based devices. Even, the untreated devices also showed very 
good reproducibility, consistent with our previous work [30]. Overall, it is concluded that the OSCs showed very good performance as well as the reproducibility with minor variation in the $\mathrm{J}_{\mathrm{sc}}, \mathrm{V}_{\mathrm{oc}}$, FF and PCE. Moreover, the device with the 10 min UVO treatment of GO/PEDOT:PSS bilayer exhibited the most optimum performance in terms of efficiency and reproducibility with smallest variations in the performance parameters when it is reproduced. It is attributed to the synergistic hybridization of GO and PEDOT:PSS in the bilayer configuration treated with the optimized (10 min) UVO exposure which eventually increased conductivity of GO (by moderately reducing it) and WF of PEDOT:PSS. Further, it is also attributed to the excellent surface morphology of HTL that was retained after 10 min UVO treatment and RMS surface roughness had insignificant increase. The excellent surface quality of HTL will definitely facilitate the extraction of charge carriers by suppressing the $\mathrm{R}_{\mathrm{S}}$ as evident in our results too. In addition, the bilayer structure also facilitates the charge carriers' extraction via hoping due to well aligned energy levels of GO/PEDOT:PSS with the donor PCDTBT. However, further improved methods for realizing the uniform and smooth GO/PEDOT:PSS films could be a facile way to improve both the efficiency and the reproducibility. 
(a)

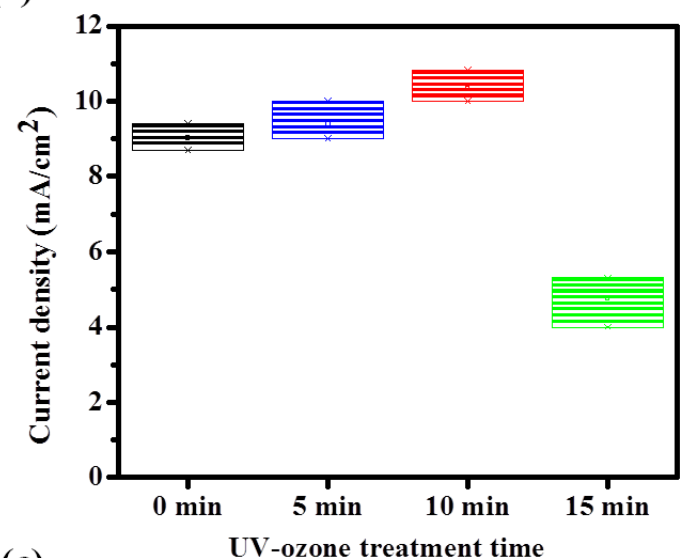

(c)

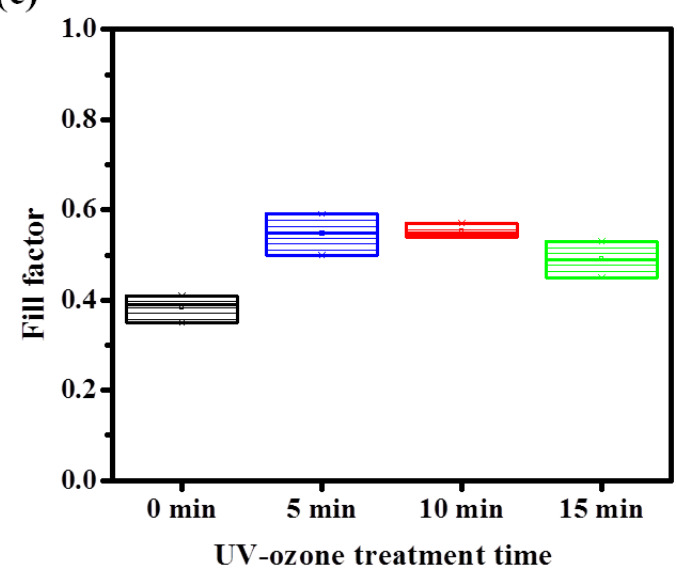

(b)

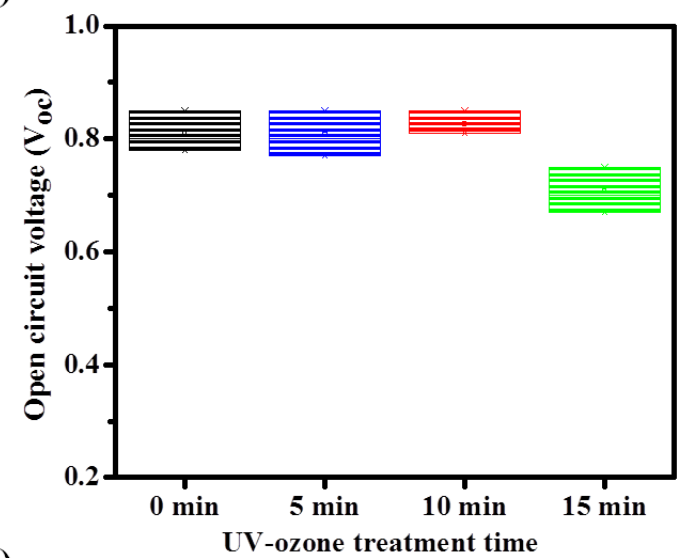

(d)

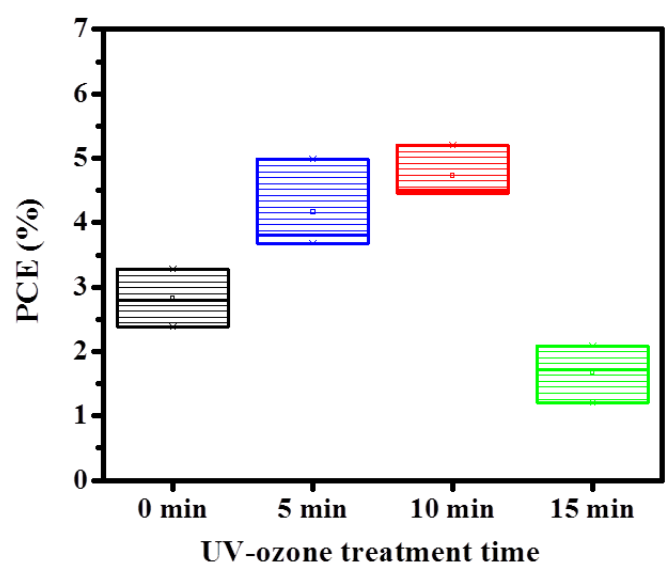

Figure 7: Reproducibility evaluation in terms of (a) $\mathrm{J}_{\mathrm{sc}}$, (b) $\mathrm{V}_{\mathrm{oc}}$ (c) FF, and (d) PCE of OSCs as a function of time under UVO treatment.

\subsection{Mechanistic Study on the Enhanced Cell Performance Due to UVO Treated HTLs}

It is reported that UVO treatment is one of the essential processes to obtain high WF for efficient hole extraction while using ITO as anode, that could be potentially performed on various buffer layers, including metal oxides, and in particular the PEDOT:PSS, to optimize the WF [35, $58,59]$. Moreover, it is also an efficient approach to enhance affectivity of GO by reducing GO for its realization as an HTL[39]. 
The oxygenated functional groups in the GO sheets possess lower conductivity and very high charge traps compared to $\mathrm{r}-\mathrm{GO}$ sheets $[33,60]$. The origin of charge trapping in GO is probably attributed to the contributions from both the structural defects i.e., vacancy or interstitial site of GO sheets and insulating gap of oxidized GO domains [60-62]. It is obvious that the oxygen functional groups, fostering the insulating properties of GO films, had been efficiently removed after UVO treatment, causing almost similar phenomenon that occur while reducing the GO [27]. In fact, the ozone molecules have the tendency to moderately reduce GO, which will lead to an enhanced conductivity due to partial conversion of GO into highly conductive graphene [39]. This fact has also been simulated and the resultant model (Figure S1, S2) clearly demonstrates the bandgap reduction of GO after removal of oxygen content which will definitely increase the conductivity and ultimately the performance of OSCs. GO, owing to its insulating characteristics, does not possess very high efficiency. In our recent work, the devices with pristine single GO HTL showed low PCEs typically below $3 \%[30,40]$ as compared to UVO treated GO only device (Table 2) that shows a PCE of $4.42 \%$. The observed increase in PCE is approximately in direct proportion to the bandgap reduction of UVO treated r-GO (Supplementary information). Hence the simulation and experimental results are very much complimenting each other as well as prove the effectiveness of the current approach. Moreover, the reduction in the oxygen content is also associated with a decrease in sheet resistance [63]. Also, the r-GO is reported to possess homogeneous film surfaces, better conductivity, an improved FF as well as the $\mathrm{J}_{\mathrm{sc}}$ and the enhanced device stability, as evident in our results too $[64,65]$. Therefore, it indicates that a facile UVO treatment of solution processed GO can moderately reduce GO to facilitate the transportation of charge carriers efficiently. 
The UVO treatment, similar to oxygen plasma treatment, is probably a general method for all PEDOT derivatives, as reported [42, 66]. Helander et al. [50]. in their study on the effect of UVO on the PEDOT:PSS reported that the enhanced WF after UVO treatment is ascribed to a metastable surface dipole, thus improves the holes extraction from the photoactive layer [67]. The Ethylenedioxythiophene group when exposed to UVO, it produces carboxyl groups caused by the de-polymerization process. Owing to the negative charge of the carboxyl group, it acts in the surface as a surface dipole, thus, the WF shifts to the higher values [37]. The increased WF improves the contact conditions between the photoactive layer and the HTL, consequently, enhances the extraction efficiency of photogenerated holes and limits the recombination of charge carriers in the photoactive blend [37]. Moreover, it is noteworthy that the short time UVO treatment effectively suppresses the reverse injection current, which reduces the injection barrier and increases the electron-blocking ability of PEDOT:PSS [66]. As a results, the $\mathrm{J}_{\mathrm{sc}}$, the FF, and hence, the PCE are improved in the OSCs. However, when the UVO treatment time is increased to $15 \mathrm{~min}$, the performance of OSCs drastically decreased. This is ascribed to the lower WF, a substantial increase in the $R_{s}$ (Figure 7d), and a significant drop in the $J_{s c}$ and the $V_{o c}$ of the corresponding device. While, a considerable drop in the FF of the device has also been observed. No further improvement beyond 10 min treatment is attributed to a couple of factors. Firstly, it is probably the saturation limit for the further improvement in the WF, which primarily started to drop after longer treatment times [42, 68]. Secondly, the surface etching caused by the UVO treatment suggests the partial removal of PEDOT:PSS, thinning of the HTL and formation of agglomerates with increased probability of film rupture and pinhole defects, which are contributing towards the performance decay $[53,66]$. Regarding the GO, the longer UVO treatment can 
probably re-oxidize the graphene particles, thus forming new GO again and hence, reducing the conductivity that will ultimately affect the device performance [39].

\subsubsection{Synergistically improved performance of the OSCs using bilayer structure}

In our previous results, UVO reduction of GO as a single HTL material enhanced the device performances of OSCs [38]. Similarly, several reports confirm the effectiveness of UVO treated PEDOT:PSS HTLs. Synergistic hybridization of UVO treated GO and PEDOT:PSS in bilayer structure offers several potential advantages. Firstly, the well matched WF of GO and PEDOT:PSS facilitates the transportation of charge carriers, increases the charge carriers mobility and causes an overall reduction of $\mathrm{R}_{\mathrm{s}}$, as evident in our current work and our previously reported results [30]. Secondly, GO underneath the PEDOT:PSS acts as a barrier against the indium diffusion of ITO. Also, it should be noted from the performance comparison of GO/PEDOT:PSS with that of single PEDOT:PSS or GO HTLs as shown in Figure 6 and Table 2, the GO/PEDOT:PSS HTL outperformed either of the individual PEDOT:PSS or GO due to a considerable increase in the photovoltaic performance parameters. Such increase may be attributed to the improved hole transportation $\left(\mathrm{J}_{\mathrm{sc}}\right)$ due to $2 \mathrm{D}$ nature of $\mathrm{GO}$, improvement in conductivity due to the formation of r-GO (Increased FF), ability of r-GO to reduce the charge carriers recombination and the increased WF (as mentioned above) of PEDOT:PSS [24]. Furthermore, as shown in Figure 6b, the stability of UVO treated r-GO HTL (Either single r-GO or r-GO underneath PEDOT:PSS) is much higher than conventional PEDOT:PSS, suggesting that r-GO functions as more stable HTL and also has a more efficient passivation property against oxygen and moisture as compared to the PEDOT:PSS [33, 69]. Hence, UVO-treated GO/PEDOT:PSS bilayer HTL can successfully compliment the drawbacks of single GO and conventional PEDOT:PSS[19]. Finally, it is important to note that the presented technique ensures the low temperature, low cost and facile fabrication, since it is 
applicable to flexible and light-weight substrates and therefore, compatible with the roll to roll production modules.

\section{CONCLUSIONS}

In conclusion, UVO treated GO/PEDOT:PSS bilayer structure have been tested as an HTL for high-efficiency and high-stability solution processable OSCs. The results obtained indicate that the optimized treatment of GO and PEDOT:PSS with UVO led to remarkably improved device performance over and above those observed for devices containing untreated or over-treated HTLs, indicating the beneficial nature of such treatment in this complementary system for the hole extraction. In particular, dramatically enhanced performance of OSCs was demonstrated by introducing 10 min treated HTL in PCDTBT:PC ${ }_{71}$ BM based HTL exhibiting 5.24\% PCE, which is also noted to be far superior to those of untreated devices. This improvement is observed to be driven primarily through enhanced conductivity of graphene after reduction/removal of oxygen groups from GO, and an enhanced WF of PEDOT:PSS due to UVO treatment. Further, it is also ascribed to improved $\mathrm{J}_{\mathrm{sc}}$ and $\mathrm{FF}$ approaching $10.82 \mathrm{~mA} / \mathrm{cm}^{2}$ and $57 \%$, respectively, for $10 \mathrm{~min}$ treated sample which indicates an effective charge extraction process and an improved contact

conditions between the photoactive layer and the HTL. Furthermore, in the stability and reproducibility tests, the devices with optimized UVO treatment (5 and $10 \mathrm{~min}$ ) of HTLs manifested much better stability. Combined with the added advantage of solution processability, these results clearly indicate that UVO treated GO and PEDOT:PSS are a promising interfacial materials in bilayer HTL structure, thus further fostering the realization of facile, highly efficient, stable, and low-cost OSCs by high-throughput roll-to-roll manufacturing. 


\section{ACKNOWLEDGMENTS}

This work is supported by High Impact Research (HIR) Grant UM.S/625/3/HIR/MoE/SC/26 with account number UM.0000080/HIR.C3 from the Ministry of Education, Malaysia. The financial support from Postgraduate Research Grant PPP, University of Malaya, with Grant Number PG1072015B is also acknowledged. We would also like to thank the Solar Photovoltaic Academic Research Consortium II (SPARC II) project, funded by WEFO for support.

\section{REFERENCES}

[1] S. Rafique, S.M. Abdullah, K. Sulaiman, M. Iwamoto, Layer by layer characterisation of the degradation process in PCDTBT: PC71BM based normal architecture polymer solar cells, Organic Electronics, 40 (2017) 65-74.

[2] S. Rafique, S.M. Abdullah, W.E. Mahmoud, A.A. Al-Ghamdi, K. Sulaiman, Stability enhancement in organic solar cells by incorporating V 2 O 5 nanoparticles in the hole transport layer, RSC Advances, 6 (2016) 50043-50052.

[3] G. Yu, J. Gao, J.C. Hummelen, F. Wudl, A.J. Heeger, Polymer photovoltaic cells: enhanced efficiencies via a network of internal donor-acceptor heterojunctions, Science, 270 (1995) 1789-1791.

[4] H. Ma, H.L. Yip, F. Huang, A.K.Y. Jen, Interface engineering for organic electronics, Advanced Functional Materials, 20 (2010) 1371-1388.

[5] B. Cao, X. He, C.R. Fetterly, B.C. Olsen, E.J. Luber, J.M. Buriak, Role of Interfacial Layers in Organic Solar Cells: Energy Level Pinning Versus Phase Segregation, ACS applied materials \& interfaces, 8 (2016) 18238-18248. 
[6] J. Lian, Y. Yuan, E. Peng, J. Huang, Interfacial layers in organic solar cells, in: Organic and Hybrid Solar Cells, Springer, 2014, pp. 121-176.

[7] R. Steim, F.R. Kogler, C.J. Brabec, Interface materials for organic solar cells, Journal of Materials Chemistry, 20 (2010) 2499-2512.

[8] L. Lu, T. Zheng, Q. Wu, A.M. Schneider, D. Zhao, L. Yu, Recent advances in bulk heterojunction polymer solar cells, Chemical reviews, 115 (2015) 12666-12731.

[9] X. Bulliard, S.G. Ihn, S. Yun, Y. Kim, D. Choi, J.Y. Choi, M. Kim, M. Sim, J.H. Park, W. Choi, Enhanced performance in polymer solar cells by surface energy control, Advanced Functional Materials, 20 (2010) 4381-4387.

[10] H. Luo, X. Lin, X. Hou, L. Pan, S. Huang, X. Chen, Efficient and air-stable planar perovskite solar cells formed on graphene-oxide-modified PEDOT: PSS hole transport layer, NanoMicro Letters, 9 (2017) 39.

[11] W. Kim, N. Kim, J.K. Kim, I. Park, Y.S. Choi, D.H. Wang, H. Chae, J.H. Park, Polymer bulk heterojunction solar cells with PEDOT: PSS bilayer structure as hole extraction layer, ChemSusChem, 6 (2013) 1070-1075.

[12] B. Ecker, J.C. Nolasco, J. Pallarés, L.F. Marsal, J. Posdorfer, J. Parisi, E. von Hauff, Degradation effects related to the hole transport layer in organic solar cells, Advanced Functional Materials, 21 (2011) 2705-2711.

[13] V. Shrotriya, G. Li, Y. Yao, C.-W. Chu, Y. Yang, Transition metal oxides as the buffer layer for polymer photovoltaic cells, Applied Physics Letters, 88 (2006) 073508.

[14] J.J. Berry, N.E. Widjonarko, B.A. Bailey, A.K. Sigdel, D.S. Ginley, D.C. Olson, Surface treatment of $\mathrm{NiO}$ hole transport layers for organic solar cells, IEEE Journal of Selected Topics in Quantum Electronics, 16 (2010) 1649-1655. 
[15] S. Lattante, Electron and hole transport layers: their use in inverted bulk heterojunction polymer solar cells, Electronics, 3 (2014) 132-164.

[16] P. Schulz, S.R. Cowan, Z.L. Guan, A. Garcia, D.C. Olson, A. Kahn, NiOX/MoO3 Bi-Layers as Efficient Hole Extraction Contacts in Organic Solar Cells, Advanced Functional Materials, 24 (2014) 701-706.

[17] R. Po, C. Carbonera, A. Bernardi, N. Camaioni, The role of buffer layers in polymer solar cells, Energy \& Environmental Science, 4 (2011) 285-310.

[18] J.-H. Huang, D. Kekuda, C.-W. Chu, K.-C. Ho, Electrochemical characterization of the solvent-enhanced conductivity of poly (3, 4-ethylenedioxythiophene) and its application in polymer solar cells, Journal of Materials Chemistry, 19 (2009) 3704-3712.

[19] D.-Y. Lee, S.-I. Na, S.-S. Kim, Graphene oxide/PEDOT: PSS composite hole transport layer for efficient and stable planar heterojunction perovskite solar cells, Nanoscale, 8 (2016) 15131522.

[20] D. Wang, N.K. Elumalai, M.A. Mahmud, M. Wright, M.B. Upama, K.H. Chan, C. Xu, F. Haque, G. Conibeer, A. Uddin, V2O5-PEDOT: PSS bilayer as hole transport layer for highly efficient and stable perovskite solar cells, Organic Electronics, 53 (2018) 66-73.

[21] Y. Wang, Q. Luo, N. Wu, Q. Wang, H. Zhu, L. Chen, Y.-Q. Li, L. Luo, C.-Q. Ma, Solutionprocessed MoO3: PEDOT: PSS hybrid hole transporting layer for inverted polymer solar cells, ACS applied materials \& interfaces, 7 (2015) 7170-7179.

[22] H.S. Dehsari, E.K. Shalamzari, J.N. Gavgani, F.A. Taromi, S. Ghanbary, Efficient preparation of ultralarge graphene oxide using a PEDOT: PSS/GO composite layer as hole transport layer in polymer-based optoelectronic devices, RSC Advances, 4 (2014) 55067-55076. 
[23] L. Zhou, D. Yang, W. Yu, J. Zhang, C. Li, An efficient polymer solar cell using graphene oxide interface assembled via layer-by-layer deposition, Organic Electronics, 23 (2015) 110115.

[24] E. Stratakis, K. Savva, D. Konios, C. Petridis, E. Kymakis, Improving the efficiency of organic photovoltaics by tuning the work function of graphene oxide hole transporting layers, Nanoscale, 6 (2014) 6925-6931.

[25] S.-S. Li, K.-H. Tu, C.-C. Lin, C.-W. Chen, M. Chhowalla, Solution-processable graphene oxide as an efficient hole transport layer in polymer solar cells, ACS nano, 4 (2010) 31693174.

[26] C.T. Smith, R.W. Rhodes, M.J. Beliatis, K. Imalka Jayawardena, L.J. Rozanski, C.A. Mills, S.R. P. Silva, Graphene oxide hole transport layers for large area, high efficiency organic solar cells, Applied Physics Letters, 105 (2014) 129_121.

[27] Y.-J. Jeon, J.-M. Yun, D.-Y. Kim, S.-I. Na, S.-S. Kim, Moderately reduced graphene oxide as hole transport layer in polymer solar cells via thermal assisted spray process, Applied Surface Science, 296 (2014) 140-146.

[28] J.C. Yu, J.I. Jang, B.R. Lee, G.-W. Lee, J.T. Han, M.H. Song, Highly efficient polymer-based optoelectronic devices using PEDOT: PSS and a GO composite layer as a hole transport layer, ACS applied materials \& interfaces, 6 (2014) 2067-2073.

[29] Z. Ding, Z. Hao, B. Meng, Z. Xie, J. Liu, L. Dai, Few-layered graphene quantum dots as efficient hole-extraction layer for high-performance polymer solar cells, Nano Energy, 15 (2015) 186-192. 
[30] S. Rafique, S.M. Abdullah, M.M. Shahid, M.O. Ansari, K. Sulaiman, Significantly improved photovoltaic performance in polymer bulk heterojunction solar cells with graphene oxide/PEDOT: PSS double decked hole transport layer, Scientific reports, 7 (2017) 39555.

[31] Y. Park, K. Soon Choi, S. Young Kim, Graphene oxide/PEDOT: PSS and reduced graphene oxide/PEDOT: PSS hole extraction layers in organic photovoltaic cells, physica status solidi (a), 209 (2012) 1363-1368.

[32] G. Eda, C. Mattevi, H. Yamaguchi, H. Kim, M. Chhowalla, Insulator to semimetal transition in graphene oxide, The Journal of Physical Chemistry C, 113 (2009) 15768-15771.

[33] J.M. Yun, J.S. Yeo, J. Kim, H.G. Jeong, D.Y. Kim, Y.J. Noh, S.S. Kim, B.C. Ku, S.I. Na, Solution-processable reduced graphene oxide as a novel alternative to PEDOT: PSS hole transport layers for highly efficient and stable polymer solar cells, Advanced Materials, 23 (2011) 4923-4928.

[34] Z.-L. Tseng, L.-C. Chen, C.-H. Chiang, S.-H. Chang, C.-C. Chen, C.-G. Wu, Efficient inverted-type perovskite solar cells using UV-ozone treated MoOx and WOx as hole transporting layers, Solar Energy, 139 (2016) 484-488.

[35] Q.V. Le, T.P. Nguyen, S.Y. Kim, UV/ozone-treated WS2 hole-extraction layer in organic photovoltaic cells, physica status solidi (RRL)-Rapid Research Letters, 8 (2014) 390-394.

[36] Y. Meng, Z. Hu, N. Ai, Z. Jiang, J. Wang, J. Peng, Y. Cao, Improving the stability of bulk heterojunction solar cells by incorporating pH-neutral PEDOT: PSS as the hole transport layer, ACS applied materials \& interfaces, 6 (2014) 5122-5129.

[37] Z. Su, L. Wang, Y. Li, H. Zhao, B. Chu, W. Li, Ultraviolet-ozone-treated PEDOT: PSS as anode buffer layer for organic solar cells, Nanoscale research letters, 7 (2012) 465. 
[38] S. Rafique, S.M. Abdullah, J. Iqbal, A. Jilani, S. Vattamkandathil, M. Iwamoto, Moderately reduced graphene oxide via UV-ozone treatment as hole transport layer for high efficiency organic solar cells, Organic Electronics, 59 (2018) 140-148.

[39] Y. Xia, Y. Pan, H. Zhang, J. Qiu, Y. Zheng, Y. Chen, W. Huang, Graphene Oxide by UVOzone Treatment as an Efficient Hole Extraction Layer for Highly Efficient and Stable Polymer Solar Cells, ACS applied materials \& interfaces, 9 (2017) 26252-26256.

[40] S. Rafique, S.M. Abdullah, H. Alhummiany, M.S. Abdel-Wahab, J. Iqbal, K. Sulaiman, Bulk Heterojunction organic solar cells with Graphene oxide hole transport layer: effect of varied concentration on photovoltaic performance, The Journal of Physical Chemistry C, 121 (2016) $140-146$.

[41] D. Yang, L. Zhou, L. Chen, B. Zhao, J. Zhang, C. Li, Chemically modified graphene oxides as a hole transport layer in organic solar cells, Chemical Communications, 48 (2012) 80788080.

[42] C. Tengstedt, A. Kanciurzewska, M.P. de Jong, S. Braun, W.R. Salaneck, M. Fahlman, Ultraviolet light-ozone treatment of poly (3, 4-ethylenedioxy-thiophene)-based materials resulting in increased work functions, Thin Solid Films, 515 (2006) 2085-2090.

[43] A.R. bin Mohd Yusoff, H.P. Kim, J. Jang, High performance organic photovoltaics with zinc oxide and graphene oxide buffer layers, Nanoscale, 6 (2014) 1537-1544.

[44] Y.-J. Jeon, J.-M. Yun, D.-Y. Kim, S.-I. Na, S.-S. Kim, High-performance polymer solar cells with moderately reduced graphene oxide as an efficient hole transporting layer, Solar Energy Materials and Solar Cells, 105 (2012) 96-102. 
[45] X. Zheng, Y. Peng, Y. Yang, J. Chen, H. Tian, X. Cui, W. Zheng, Hydrothermal reduction of graphene oxide; effect on surface-enhanced Raman scattering, Journal of Raman Spectroscopy, 48 (2017) 97-103.

[46] J. Paredes, S. Villar-Rodil, P. Solís-Fernández, A. Martínez-Alonso, J. Tascon, Atomic force and scanning tunneling microscopy imaging of graphene nanosheets derived from graphite oxide, Langmuir, 25 (2009) 5957-5968.

[47] Y.-J. Noh, S.-C. Park, I.-T. Hwang, J.-H. Choi, S.-S. Kim, C.-H. Jung, S.-I. Na, Highperformance polymer solar cells with radiation-induced and reduction-controllable reduced graphene oxide as an advanced hole transporting material, Carbon, 79 (2014) 321-329.

[48] P. Wang, Z.-G. Liu, X. Chen, F.-L. Meng, J.-H. Liu, X.-J. Huang, UV irradiation synthesis of an Au-graphene nanocomposite with enhanced electrochemical sensing properties, Journal of Materials Chemistry A, 1 (2013) 9189-9195.

[49] S.M. Hafiz, R. Ritikos, T.J. Whitcher, N.M. Razib, D.C.S. Bien, N. Chanlek, H. Nakajima, T. Saisopa, P. Songsiriritthigul, N.M. Huang, A practical carbon dioxide gas sensor using room-temperature hydrogen plasma reduced graphene oxide, Sensors and Actuators B: Chemical, 193 (2014) 692-700.

[50] M. Helander, Z. Wang, M. Greiner, Z. Liu, K. Lian, Z. Lu, The effect of UV ozone treatment on poly (3, 4-ethylenedioxythiophene): poly (styrenesulfonate), Applied Physics Letters, 95 (2009) 280 .

[51] D. Briggs, G. Beamson, XPS studies of the oxygen 1s and 2s levels in a wide range of functional polymers, Analytical Chemistry, 65 (1993) 1517-1523. 
[52] A.A. Farah, S.A. Rutledge, A. Schaarschmidt, R. Lai, J.P. Freedman, A.S. Helmy, Conductivity enhancement of poly (3, 4-ethylenedioxythiophene)-poly (styrenesulfonate) films post-spincasting, Journal of Applied Physics, 112 (2012) 113709.

[53] T. Nagata, S. Oh, T. Chikyow, Y. Wakayama, Effect of UV-ozone treatment on electrical properties of PEDOT: PSS film, Organic Electronics, 12 (2011) 279-284.

[54] G. Greczynski, T. Kugler, W. Salaneck, Characterization of the PEDOT-PSS system by means of X-ray and ultraviolet photoelectron spectroscopy, Thin Solid Films, 354 (1999) 129135.

[55] E. Britannica, Encyclopædia britannica, Chicago: Common Law, (2009).

[56] E. Vitoratos, S. Sakkopoulos, E. Dalas, N. Paliatsas, D. Karageorgopoulos, F. Petraki, S. Kennou, S.A. Choulis, Thermal degradation mechanisms of PEDOT: PSS, Organic Electronics, 10 (2009) 61-66.

[57] H. Alhummiany, S. Rafique, K. Sulaiman, XPS Analysis of the Improved Operational Stability of Organic Solar Cells Using a V2O5 and PEDOT: PSS Composite Layer: Effect of Varied Atmospheric Conditions, The Journal of Physical Chemistry C, 121 (2017) 7649-7658.

[58] F. Wang, G. Sun, C. Li, J. Liu, S. Hu, H. Zheng, Z.a. Tan, Y. Li, Finding the lost open-circuit voltage in polymer solar cells by UV-ozone treatment of the nickel acetate anode buffer layer, ACS applied materials \& interfaces, 6 (2014) 9458-9465.

[59] H.-W. Lu, P.-C. Kao, Y.-D. Juang, S.-Y. Chu, The effects of ultraviolet-ozone-treated ultrathin $\mathrm{MnO}$-doped $\mathrm{ZnO}$ film as anode buffer layer on the electrical characteristics of organic light-emitting diodes, Journal of Applied Physics, 118 (2015) 185501.

[60] S. Wang, J. Pu, D.S. Chan, B.J. Cho, K.P. Loh, Wide memory window in graphene oxide charge storage nodes, Applied Physics Letters, 96 (2010) 143109. 
[61] T.-W. Kim, Y. Gao, O. Acton, H.-L. Yip, H. Ma, H. Chen, A.K.-Y. Jen, Graphene oxide nanosheets based organic field effect transistor for nonvolatile memory applications, Applied Physics Letters, 97 (2010) 147.

[62] J.-H. Chen, W. Cullen, C. Jang, M. Fuhrer, E. Williams, Defect scattering in graphene, Physical review letters, 102 (2009) 236805.

[63] Y.-L. Huang, H.-W. Tien, C.-C.M. Ma, S.-Y. Yang, S.-Y. Wu, H.-Y. Liu, Y.-W. Mai, Effect of extended polymer chains on properties of transparent graphene nanosheets conductive film, Journal of Materials Chemistry, 21 (2011) 18236-18241.

[64] H.P. Kim, A.R. bin Mohd Yusoff, J. Jang, Organic solar cells using a reduced graphene oxide anode buffer layer, Solar Energy Materials and Solar Cells, 110 (2013) 87-93.

[65] X. Cheng, J. Long, R. Wu, L. Huang, L. Tan, L. Chen, Y. Chen, Fluorinated Reduced Graphene Oxide as an Efficient Hole-Transport Layer for Efficient and Stable Polymer Solar Cells, ACS Omega, 2 (2017) 2010-2016.

[66] W. Cai, C. Musumeci, F.N. Ajjan, Q. Bao, Z. Ma, Z. Tang, O. Inganäs, Self-doped conjugated polyelectrolyte with tuneable work function for effective hole transport in polymer solar cells, Journal of Materials Chemistry A, 4 (2016) 15670-15675.

[67] H.K. Lee, J.-K. Kim, O.O. Park, Effects of UV light-irradiated buffer layer on the performance of polymer solar cells, Organic Electronics, 10 (2009) 1641-1644.

[68] A. Benor, S.-y. Takizawa, C. Pérez-Bolívar, P. Anzenbacher Jr, Efficiency improvement of fluorescent OLEDs by tuning the working function of PEDOT: PSS using UV-ozone exposure, Organic Electronics, 11 (2010) 938-945.

[69] H. Kim, Y. Miura, C.W. Macosko, Graphene/polyurethane nanocomposites for improved gas barrier and electrical conductivity, Chemistry of Materials, 22 (2010) 3441-3450. 
